\title{
Anticoncepción de Emergencia: Autonomía de las Adolescentes y Derechos de sus Padres
}

Este comentario, junto a los documentos que analiza, está disponible en www.anuariocdh.uchile.cl

\author{
Verónica Undurraga Valdés*
}

\section{Introducción}

$\angle$ a anticoncepción de emergencia ha sido motivo de intensos debates jurídicos en Chile a partir del año 2001. Hasta la fecha, y después de siete sentencias recaídas en el tema ${ }^{1}$, no existe una determinación judicial clara sobre el estatus jurídico del embrión ni sobre el alcance del deber constitucional de proteger la vida del que está por nacer, dos aspectos centrales de la controversia ${ }^{2}$.

Sin perjuicio de ello, el debate ha adquirido una nueva arista a partir de la dictación de la resolución № 584 del Ministerio de Salud (2006), que estableció, entre otras medidas, la distribución del anticonceptivo de emergencia a personas mayores de 14 años en el sistema público de salud, sin que para ello sea necesaria la autorización de sus padres ${ }^{3}$. Así, quienes recientemente han recurrido a los tribunales con la intención de impedir la comercialización y la distribución de la anticoncepción de emergencia, afirman que el contenido de la resolución mencionada vulneraría el derecho preferente de los padres de educar a sus hijos (artículo 19 № 10 inciso 3 de la Constitución Política).

La Corte de Apelaciones de Santiago, fallando un recurso de protección, negó que la decisión ministerial afectara este derecho de los padres. De acuerdo a la Corte:

"[l]os padres (...) siempre podrán educar a sus hijos en las cuestiones de la sexualidad y la regulación de la fertilidad de la manera que en su concepto les parezca adecuada, incluso expresamente podrán formarlos para que no recurran en circunstancia alguna a la ingesta del anticonceptivo de emergencia, sin que su afán de contrariar la política gubernamental pueda acarrearles consecuencia negativa alguna..." ${ }^{4}$.

Por otra parte, El Tribunal Constitucional acogió un requerimiento presentado por un grupo de diputados en contra de la mencionada resolución ${ }^{5}$, pero lo hizo por consideraciones de forma. Declaró, sin haber tenido competencia para hacerlo ${ }^{6}$, que el acto administrativo era inconstitu-

* Abogada, Universidad de Chile. Coordinadora del Programa de Mujeres y Derechos Humanos, Centro de Derechos Humanos, Facultad de Derecho, Universidad de Chile.

1 Corte de Apelaciones de Santiago, rol № 850-2001; Corte Suprema, rol № 2186-2001; $20^{\circ}$ Juzgado Civil de Santiago, rol No 5839-2004; Corte de Apelaciones de Santiago, rol No 4200-03; Corte Suprema, rol № 1039-2005; Corte de Apelaciones de Santiago, rol № 4693-2006; Tribunal Constitucional, rol № 591-06.

2 Al respecto, Bascuñán R., Antonio, "Después de la Píldora", en Anuario de Derechos Humanos 2006, Centro de Derechos Humanos, Facultad de Derecho, Universidad de Chile, 2006, p. 235.

3 Resolución exenta № 584 del Ministerio de Salud, de 1 de septiembre de 2006, que fija el texto del documento denominado "Normas Nacionales sobre Regulación de la Fertilidad".

4 Corte de Apelaciones de Santiago, rol No 4693-2006, considerando $7^{\circ}$.

5 Requerimiento de inconstitucionalidad ante el Tribunal Constitucional, presentado el día 30 de septiembre de 2006 por 31 diputados de la Alianza por Chile y un diputado independiente.

6 En nuestra opinión el Tribunal Constitucional carecía de competencia para pronunciarse sobre la constitucionalidad de una resolución. Coincidimos con el voto de minoría en que el control jurisdiccional, en este caso, correspondía a la justicia ordinaria. 
cional porque la materia regulada por la resolución debía estar contenida en un decreto supremo reglamentario suscrito por la Presidenta de la República. Por ello, es razonable prever que en el mediano plazo se impugne el decreto supremo $\mathrm{N}^{0} 48 / 07^{7}$, que reemplazó a la resolución $N^{0} 584 / 06$, y que se obligue al Tribunal Constitucional a conocer nuevamente esta materia y a pronunciarse finalmente sobre el fondo de la controversia.

Este comentario surge de constatar cómo en el debate jurídico sobre la anticoncepción de emergencia se han omitido sorprendentemente todos los argumentos que aluden a los derechos de las mujeres que serán afectados por la autorización o prohibición de acceso a ella.

Del conjunto de derechos de las mujeres que debieran considerarse a propósito de la constitucionalidad de las normas que autorizan la comercialización o distribución de la píldora, este trabajo se refiere al derecho a la autonomía y analiza su relación con el derecho preferente de los padres a educar a sus hijas adolescentes. Es importante resaltar el carácter específico de este trabajo, al limitarse exclusivamente al análisis de dos derechos, entre los varios intereses jurídicos y derechos subjetivos que pueden hacerse valer en este dilema. Esta especificidad no debe interpretarse como un intento de excluir del debate general otras consideraciones, ni como un juicio respecto de la relevancia de los distintos derechos e intereses. Una adecuada decisión debiera tomar en consideración a todo el conjunto de derechos y bienes jurídicos que resulten afectados por ella.

En lo que sigue de este artículo, se analiza el contenido del derecho a la autonomía (II); se discuten las dificultades que tiene el ejercicio de la autonomía para las mujeres, especialmente las jóvenes y las que se encuentran en situación de pobreza, y se explica cómo el género, la edad y la vulnerabilidad social son factores decisivos en la determinación del impacto que tiene sobre las personas una decisión pública sobre la anticoncepción de emergencia (III); se reflexiona sobre el significado de la autonomía en el contexto de la experiencia de las mujeres durante el embarazo y en sus relaciones de familia (IV); y finalmente se expone la tesis de que el derecho y deber de los padres de educar a sus hijas supone la preparación de las jóvenes en la adquisición de capacidades para ejercer de manera progresiva, y de acuerdo a su madurez, el derecho de autonomía que les reconoce nuestro sistema constitucional y específicamente la Convención sobre los Derechos del Niño ${ }^{8}(\mathrm{~V})$.

\section{Autonomía}

La autonomía personal, libertad que tiene cada individuo para actuar conforme a su propia concepción acerca de lo que es valioso, necesario, deseable o correcto, es un valor fundamental en nuestra comprensión actual de la moral y la política. La autonomía puede considerarse un valor intrínseco si el hecho de ser un agente de la propia vida se aprecia como un bien en sí mismo, y no sólo por sus resultados o consecuencias. Sin embargo, también puede verse como un valor instrumental, si se justifica en el argumento que establece que nadie está en mejor posición para determinar lo que es una vida buena, más que la persona llamada a vivirla.

La autonomía tiene una dimensión fáctica y una normativa. En la práctica, nadie goza de una plena autonomía. Es más realista pensar que casi siempre escogemos reforzando decisiones ya definidas por quienes nos precedieron y por nuestra comunidad de referencia. Sin embargo, en la posibilidad siempre presente de apartarse de los condicionamientos o de asumir reflexivamente

7 DS N 48 Ministerio de Salud, de 26 de enero de 2007, publicado en el Diario Oficial el 3 de febrero de 2007.

8 Adoptada y abierta a la firma y ratificación por la Asamblea General en su resolución 44/25, de 20 de noviembre de 1989. Entrada en vigor: 2 de septiembre de 1990. Promulgada por Decreto 803 del 14 de agosto de 1990. Fecha de publicación 27 de septiembre de 1990. 
como propios los caminos ya trazados, se dan espacios de creación -de pensamientos, percepciones, expresiones y acciones- en los que se ejerce la autonomía personal. Somos autónomos cuando nuestros actos regularmente reflejan valores, compromisos, principios o preferencias que son significativos para nosotros, de manera tal que expresan nuestra identidad.

El ejercicio de la autonomía requiere de ciertas competencias personales, entre ellas una mínima capacidad de reflexión y de conexión con los propios sentimientos; y de algunas condiciones básicas, como tener alternativas entre las que optar y cierto grado de libertad frente a la interferencia de terceros. Sin embargo, no obstante la deficiencia en esas capacidades o condiciones, atribuimos normativamente una autonomía básica a todas las personas adultas para reconocerles igual estatus moral y político. Los sistemas políticos basados en principios liberales suponen, aun a costa de aceptar una ficción, un modelo de persona autónoma. El sistema se legitima porque sus principios y normas son aceptados, o al menos están en condiciones de ser aceptados, por cada una de las personas a quienes rige ${ }^{9}$.

\section{Autonomía cuesta arriba: mujeres, mujeres jóvenes y mujeres jóvenes en situación de pobreza}

En la evolución de nuestras concepciones morales, políticas y jurídicas hacia formas de mayor igualdad, las mujeres han ido "acomodándose" con dificultad a categorías que fueron creadas antes de que se concibiera que ellas podrían ser seres morales plenos, ciudadanas y sujetos de derecho. Esas categorías, entre ellas los derechos fundamentales, reflejan inevitablemente imaginaciones y experiencias masculinas de la realidad, en que las mujeres se reconocen sólo cuando esas imaginaciones y experiencias son compartidas por los dos géneros. Aquella parte de la percepción y vivencia del mundo que es exclusivamente femenina (como el embarazo, y hasta hace poco la crianza) no se considera para definir el contenido de las categorías. En la medida que las mujeres se alejan más del modelo masculino que sirvió para definir los derechos fundamentales, entre ellos la autonomía, más difícil se les hace la titularidad de estos derechos.

Por otro lado, el sujeto autónomo es típicamente adulto. Los adolescentes se encuentran en una situación intermedia entre la dependencia de los niños y la total autonomía de los adultos. Las mujeres adolescentes deben, por lo tanto, agregar a las dificultades que le impone su género, aquellas que le impone su edad y que implican la afirmación de grados cada vez mayores de autonomía.

Por último, la situación de pobreza ha sido históricamente sinónimo de vulnerabilidad frente a los actos de poder del Estado y de terceros. Una mujer, que además de ser adolescente es pobre, tendrá una carga adicional a la hora de reclamar el respeto de su autonomía.

En las siguientes líneas se hace un bosquejo elemental y necesariamente incompleto, que explica cómo el género, la edad y la situación de pobreza son aspectos relevantes para entender el impacto que una decisión pública sobre la anticoncepción de emergencia puede tener en el ejercicio de la autonomía personal.

\section{Mujeres}

No es casual que en los escritos y sentencias sobre la anticoncepción de emergencia, las mujeres que tomarían la píldora aparezcan siempre aludidas como "las madres" (en los argumentos sobre la protección de la vida del embrión) o "las hijas" (en los argumentos sobre el derecho de

9 Friedman, M., Autonomy, Gender, Politics, Oxford University Press, New York, 2003, pp. 163 y ss. 
los padres a educar a sus hijos). Esta forma referencial de hablar de las mujeres revela lo que nuestra cultura entiende que son la identidad y el rol femeninos. Las mujeres son seres que viven para otros. Ellas están abiertas a las necesidades físicas y emocionales de los demás, se definen a partir de los vínculos que forman con quienes se relacionan. El reconocimiento de su dignidad se funda, demasiadas veces $-y$ con el riesgo que esto implica-, en el papel que cumplen en la vida de otros.

Esta imagen de las mujeres, sin embargo, se contrapone abiertamente con dos nociones que aparecen imbricadas y a veces se confunden con la autonomía como autodeterminación: la libertad negativa y la privacidad.

La libertad negativa supone un área delimitada dentro del cual una persona puede actuar sin ser obstruida o interferida por otros ${ }^{10}$. Los derechos fundamentales clásicos están configurados conforme a este modelo espacial: constituyen esferas protegidas de la interferencia estatal. El derecho además asegura que terceros no afecten ese ámbito mínimo de libertad individual reconocido a cada persona. Sin embargo, la concepción de la feminidad como la natural predisposición a estar abiertos y disponibles frente a las necesidades de los demás, transforma a las mujeres en sujetos poco aptos para el ejercicio de derechos fundamentales, si se entienden éstos solamente como esferas de exclusión. Esta concepción de los derechos y esta particular construcción de la feminidad, han contribuido a la continua legitimación por parte de los operadores jurídicos de normas y conductas que desconocen los derechos de las mujeres, haciendo especialmente precaria la protección de su autonomía.

La privacidad, por otra parte, es un término que alude a muchas situaciones diversas, que no comparten entre ellas elementos comunes. Algunos de estos contextos están vinculados a la idea de autonomía, por lo que con frecuencia se asume erróneamente que la autonomía existe en las esferas de privacidad. La privacidad puede referirse, por ejemplo, a soledad, secreto, reclusión, prohibición de acceso, confidencialidad, intimidad, familia, sexualidad, imagen corporal, espacio propio, autonomía, control sobre la información personal, entre otras situaciones ${ }^{11}$. Sin embargo, en los espacios típicamente privados, como la sexualidad y la familia, las mujeres suelen experimentar severas restricciones a su autonomía. La muy citada definición de la privacidad como "el derecho a ser dejado solo (o en paz)"12 es antitética frente a la expectativa cultural que existe respecto de las mujeres y que supone su disponibilidad permanente, especialmente respecto de los miembros de su familia. La idea romántica del hogar como el lugar en que la persona puede libremente expresar su ser más auténtico, sin las imposiciones y deberes que exige la vida social, no se corresponde muchas veces con la vivencia femenina del espacio familiar, que para las mujeres está recargado de obligaciones y deberes impuestos por expectativas y necesidades ajenas.

Las características de protección frente a la interferencia y posibilidad de actuar reflejando las convicciones y sentimientos más propios, hermanan la libertad negativa y la privacidad con el concepto de autonomía. Sin embargo, son estas mismas conexiones -que en nuestra opinión no debieran agotar el contenido de la autonomía- las que dificultan su ejercicio por parte de las mujeres; porque se percibe la autodeterminación de ellas como una amenaza a la cohesión y solidaridad social, dos elementos que continuamente se identifican como el aporte que las mujeres hacen a la sociedad.

\footnotetext{
10 Berlin, I., "Two Concepts of Liberty", en Four Essays on Liberty, Oxford, USA, 1990, p. 118-172.

11 Undurraga, V, "La Privacidad como Bien Jurídico", en Estudios de derecho civil: Código y dogmática en el sesquicentenario de la promulgación del Código Civil. Jornadas Nacionales de Derecho Civil, Valdivia, Juan Andrés Varas Braun, Susan Turner Saelzer (Coordinadores), Lexis Nexis, 2005, p. 509.

12 Samuel D. Warren y Louis D. Brandies, "The Right to Privacy", en Harvard Law Review, 193 (1890).
} 


\section{Mujeres jóvenes}

La anticoncepción de emergencia se ofrece principalmente con el objetivo de evitar los embarazos y abortos en adolescentes ${ }^{13}$. Aunque a partir de 1998 se ha logrado disminuir la fecundidad adolescente por mayor uso de anticoncepción, la incidencia sigue siendo alta porque el riesgo de embarazo aumenta con la tendencia al inicio más temprano de la vida sexual ${ }^{14}$. Los y las jóvenes se demoran cada vez más en independizarse física y económicamente de su familia de origen. Si antes era normal que una adolescente dejara de estudiar para casarse o se embarazara e iniciara una nueva familia al terminar el colegio, ahora esa misma situación se ha transformado en un problema social, porque interrumpe su educación. Además de los muchos beneficios de la escolaridad, es necesario considerar que ésta ha reemplazado al matrimonio como la fuente de seguridad económica en que las personas confían.

No están claras las expectativas que los padres y la sociedad tienen respecto del ejercicio de la sexualidad de los y las jóvenes en los largos años que transcurren desde que inician su vida sexual hasta el tiempo en que se independizan ${ }^{15}$. Contra toda evidencia, se trata a los hijos e hijas adolescentes como si no tuvieran vida sexual y se espera particularmente de las jóvenes que respondan a ese trato no embarazándose. Las personas jóvenes con actividad sexual que intentan evitar el embarazo y las enfermedades de transmisión sexual, es decir, quienes actúan reflexivamente de acuerdo a sus circunstancias, son criticadas familiar y socialmente por el ejercicio de esa autonomía, considerada inadecuada para su edad. Las mujeres son especialmente castigadas si previenen el embarazo, porque implica que asumen disposición e iniciativa frente al sexo.

\section{Mujeres jóvenes en situación de pobreza}

La pobreza funciona muchas veces como una autorización para intervenir en la vida privada de las personas y un motivo para desconocer valor a las decisiones tomadas por quienes se encuentran inmersos en ella.

Nada impide que mujeres con medios económicos consulten confidencialmente a un médico y con una orden de éste compren en la farmacia la píldora del día después. El acceso a información, dinero y confianza, sumado al hecho de pagar por el servicio médico, otorgan poder a las mujeres frente al profesional de la salud y facilitan el ejercicio de la autonomía necesaria para lograr superar los obstáculos que encuentran para acceder a la anticoncepción de emergencia. Por el contrario, mujeres en situación de pobreza, con la misma necesidad de acceder a la anticoncepción de emergencia, probablemente deberán dar más explicaciones incómodas al médico o matrona que las atienda en el sistema público de salud, recibirán recomendaciones no solicitadas sobre el manejo de su fertilidad y tendrán menos posibilidades de reaccionar ante una intervención paternalista que les parezca ofensiva.

Por otro lado, el embarazo es una realidad que afecta desproporcionadamente a las adolescentes pobres $^{16}$. Funciona como un síntoma -más que como una causa, como regularmente se afirma- de la situación de pobreza y desesperanza ${ }^{17}$. Se embarazan más quienes tienen menos confianza en

\footnotetext{
13 Ministerio de Salud, Normas Nacionales sobre Regulación de la Fertilidad, 2006, p.78.

14 Ídem, p. 122.

15 Según datos del INE respecto del año 2000, las adolescentes y jóvenes viven aproximadamente 13 años de vida fértil antes de casarse. El promedio de inicio de la relación sexual de las jóvenes según cifras del año 2001 fue de 17,6 años. Sin embargo, el promedio para el grupo de estas jóvenes que sólo alcanzó la educación básica fue de 15,6 años. Ídem.

16 Op. cit. Nota 9, p. 122.

17 Sobre esto, ver Luker, K., Dubious Conceptions. The Politics of Teenage Pregnancy, Harvard, 1997. Estudios chilenos coinciden con lo descubierto por Luker en Estados Unidos; las adolescentes que se embarazan frecuentemente habían
} 
poder alcanzar metas personales no asociadas a la maternidad ${ }^{18}$. Para jóvenes sin expectativas, tener descendencia puede ser el único proyecto propio y significativo al que podrán acceder y volcar sus energías. Frente a eso, no son convincentes, porque no son realistas, las promesas de mejor futuro que se asocian a la decisión de postergar el embarazo. La decisión de tener un hijo (que muchas veces toma la forma de aceptar pasivamente la posibilidad de un embarazo), bajo esa lógica y circunstancias, se considera una prueba de la ineptitud de las jóvenes en situación de pobreza para ejercer su autonomía responsablemente. La crítica social, sin embargo, olvida que el problema no está en la falta de racionalidad de la decisión, sino en que ésta se dio en un contexto de tanta deprivación que la maternidad temprana fue, para esas jóvenes, una alternativa atractiva, dentro de las escasas disponibles.

Así, la pobreza no sólo disminuye la autonomía real que pueda ejercerse, sino que en la práctica opera como una razón para privar del ejercicio de la autonomía en áreas que normalmente están cubiertas por la adscripción normativa que el sistema jurídico hace. Esto ocurre cada vez que se estima que quienes viven en la pobreza no están capacitadas para tomar decisiones; y esa evaluación social respecto de las aptitudes para ejercer la autonomía no se aplica con igual celo (o simplemente no se aplica) a las personas con recursos.

\section{La mujer como sujeto}

Es necesario rescatar el valor que tiene la autonomía para las mujeres. Una persona, hombre o mujer, que se forme sus propios juicios acerca de lo que es correcto, valioso, deseable o verdadero, no puede, sin perder el respeto hacia sí misma o la noción que tiene acerca de quien ella es, renunciar a guiar su vida de acuerdo a esas convicciones. El respeto a la autonomía de las mujeres asegura su consideración como sujetos morales, capaces de discernir y responsabilizarse por sus decisiones. A la vez, obliga a que los intereses de las mujeres individuales reciban igual consideración que los de sus pares hombres en el diseño de los principios y normas que rigen a la sociedad, incluyendo la conceptualización de los propios derechos fundamentales y el contenido y alcance de los deberes constitucionales. Sólo los individuos que gozan de autonomía pueden exigir la modificación de normas y prácticas que son opresivas, porque han participado en la definición y validación de los principios de justicia que establecen los criterios que permiten calificar las normas y las prácticas.

Un ejemplo servirá para ilustrar la diferencia que en términos jurídicos tiene otorgar o no igual consideración a los intereses de las mujeres como sujetos autónomos. En un artículo publicado por Estudios Públicos ${ }^{19}$, el profesor Arturo Fermandois reconoce que el deber constitucional de proteger al que está por nacer impone a la mujer una exigencia mucho más intensa que al hombre en términos de afectación de su autonomía personal. Fermandois estima, sin embargo, que esta afectación diferenciada es legítima, porque proviene de una realidad fisiológica (sólo las mujeres se embarazan) que el derecho no está llamado a cambiar.

desertado del sistema escolar antes del embarazo. Asimismo, un 22\% de las adolescentes embarazadas afirma que "deseaba un embarazo". Ver González, E., y otros, "Estudio comparativo de factores familiares y personales en adolescentes consultantes por embarazo y anticoncepción", en Revista de la Sociedad Chilena de Obstetricia y Ginecología Infantil y de la Adolescente, Vol.5 (2), 1998; pp. 42-48.

18 ĺdem.

19 Fermandois, A., "La Píldora del Día Después: Aspectos Normativos", en Estudios Públicos, 95, Invierno, 2004. pp. 92-118. 
Es curioso, por decir lo menos, que una norma destinada a regir siempre y exclusivamente en situaciones de embarazo de una mujer se justifique en términos de un trato igualitario con el hombre 20 y que se use el argumento de que sólo la mujer se embaraza -como si fuera una excepción a la regla general de que las personas no se embarazan- para explicar y justificar por qué la intensidad de la afectación a su autonomía debe ser diferenciada, aumentada, y tolerada. ¿No habría sido razonable construir un parámetro que se relacione con la experiencia de las mujeres, y no con la de los hombres, para determinar el grado aceptable de restricción a la autonomía personal?

El profesor Fermandois entiende que las mujeres están destinadas a aceptar un grado muy intenso de afectación de su autonomía en razón de una realidad fisiológica que el derecho no puede, como es obvio, cambiar. Lo que hace interesante su argumento es que él no cuestiona el contenido y alcance que se le está dando a la norma constitucional, contenido y alcance que el derecho sí puede perfectamente cambiar, en consideración precisamente del reconocimiento y respeto a la realidad fisiológica de las mujeres.

Específicamente en el tema reproductivo, es esencial para que la autonomía tenga algún significado relevante para las mujeres, que se visibilice su propia experiencia reproductiva. En Chile, la imagen que existe de las mujeres que podrían acceder a la anticoncepción de emergencia o eventualmente a un aborto está muy distorsionada por los miedos sociales que surgen cuando las mujeres adoptan decisiones autónomas en estas materias: el miedo a que las mujeres no quieran tener más niños y disminuya la población, así como el temor de que las mujeres tomen decisiones por motivos egoístas y abandonen la responsabilidad que hasta ahora han asumido de mantener los vínculos de solidaridad familiar.

Esa desconfianza es ofensiva para las miles de mujeres cuyas motivaciones al usar la píldora o al abortar no son la desafección ni el egoísmo que se les atribuye. Ellas mayoritariamente actúan en la esfera reproductiva (en el embarazo, la anticoncepción y el aborto) incorporando en su decisión el afecto y responsabilidad que sienten hacia los miembros de su familia. Si la sociedad no se convence de eso, no es por falta de testimonios de mujeres, sino porque esos testimonios no se han tomado en serio, han pesado menos en la balanza jurídica que las aprensiones de quienes sienten que la decisión de una mujer, en materias que nadie mejor que ella conoce, es peligrosa, y debe por lo tanto ser sustituida.

Aparte de ser ofensiva, esta desconfianza es jurídicamente inaceptable. Si efectivamente las mujeres decidieran disminuir su fecundidad bajo los límites que socialmente se consideran deseables para efectos demográficos, o si las mujeres se rebelaran ante la exigencia de ser las que asumen en forma exclusiva la tarea de construir los lazos de solidaridad familiar, el derecho -por la vía de la norma o de la privación de oportunidades- no puede legítimamente obligarlas a reproducirse o a ser el sustento único de la familia en las labores físicas y afectivas de cuidado. La única respuesta no paternalista y respetuosa de la autonomía y dignidad humana en estos casos, es la de readecuar las condiciones de la vida social, de manera que los beneficios y costos asociados al trabajo reproductivo y al cuidado se repartan equitativamente entre hombres y mujeres.

Uno de los grandes desafíos que presenta el derecho a la autonomía respecto de las mujeres es el de dar sentido y preservar el valor de la autodeterminación en las relaciones de interdependencia que ellas forman especialmente con sus hijos y otros miembros de su familia ${ }^{21}$, así como en las situaciones de dependencia económica que usualmente viven mientras se dedican a la crianza,

\footnotetext{
20 Sostiene Fermandois que tanto hombre como mujer están obligados a proteger la vida del que está por nacer.

21 Sobre esta idea, ver Nedelsky, J., "Reconceiving Autonomy: Sources, Thoughts and Possibilities", en Yale Journal of Law and Feminism 1, 1989, pp. 7-36.
} 
el cuidado de personas desvalidas o a otras labores no remuneradas que aportan al bienestar general de la familia. Es necesario fomentar que las relaciones humanas promuevan la autonomía de las personas en vez de fundarse en el ejercicio de la autonomía de algunos y la anulación de la autonomía de otros. Y en lo normativo, es fundamental hacer visible a las mujeres y a sus experiencias como sujetos morales.

La circunstancia de que la anticoncepción de emergencia sea desproporcionadamente relevante para las mujeres, especialmente si son jóvenes y viven en situación de pobreza, no puede ser un dato indiferente al momento de evaluar la constitucionalidad de la norma que permite su distribución. Tanto el sujeto constitucional, como el contenido de los derechos fundamentales, en particular el de la autonomía, deben reflejar las vivencias femeninas, así como las condiciones de vulnerabilidad (producto de la inmadurez, de la pobreza y, en general, de situaciones de dependencia) que todos los seres humanos, hombres y mujeres, experimentamos en distintas etapas de nuestra vida.

\section{Derechos de los padres y autonomía de las mujeres adolescentes}

Los padres tienen el derecho preferente y el deber de educar a sus hijos. Así lo reconoce la Constitución Política ${ }^{22}$ y la Convención sobre los Derechos del Niño (CDN) ${ }^{23}$. Por otra parte, los niños, niñas y adolescentes son titulares de derechos humanos y constitucionales. La CDN reafirma este principio, contempla reglas especiales para que puedan ejercer sus derechos humanos y establece un régimen particular para su protección, teniendo en cuenta su proceso de desarrollo físico y mental.

Por ser personas en formación, los niños y niñas ejercen su derecho a la autonomía de manera progresiva, en función de su edad y madurez. Se confía preferentemente a los padres la preparación de sus hijos e hijas para la vida independiente en sociedad. El derecho de los padres implica reconocerles la potestad de definir el tiempo y las formas en que esa preparación hacia la plena autonomía se va a desarrollar. El ejercicio de este derecho no puede, sin embargo, comprometer seriamente la autonomía futura de su descendencia ${ }^{24}$. No se permite, por ejemplo, restringir absolutamente el contacto de los hijos e hijas con personas ajenas a la familia, evitar que aprendan a leer o esterilizarlos, porque estas acciones impiden ejercer la autonomía y sus otros derechos en el futuro ${ }^{25}$. Los hijos e hijas serán tratados por la sociedad como personas autónomas a partir de

22 Artículo 19 № 10: "La Constitución asegura a todas las personas... [e]l derecho a la educación. La educación tiene por objeto el pleno desarrollo de la persona en las distintas etapas de su vida. Los padres tienen el derecho preferente y el deber de educar a sus hijos. Corresponderá al Estado otorgar especial protección al ejercicio de este derecho...".

$23 \mathrm{CDN}$, Artículo 5: "Los Estados Partes respetarán las responsabilidades, los derechos y los deberes de los padres o, en su caso, de los miembros de la familia ampliada o de la comunidad, según establezca la costumbre local, de los tutores u otras personas encargadas legalmente del niño de impartirle, en consonancia con la evolución de sus facultades, dirección y orientación apropiadas para que el niño ejerza los derechos reconocidos en la presente Convención".

24 Sobre los límites a las decisiones de los padres basadas en la protección de la autonomía futura de los hijos e hijas en el campo de la salud, véase la sentencia de la Corte Constitucional de Colombia T-477/95, de la cual extracto: "En principio los padres pueden tomar ciertas decisiones en relación con el tratamiento médico de sus hijos, incluso, a veces, contra la voluntad aparente de éstos. Sin embargo, ello no quiere decir que los padres puedan tomar, a nombre de su hijo, cualquier decisión médica relativa al menor, por cuanto el niño no es propiedad de sus padres sino que él ya es una libertad y una autonomía en desarrollo, que tiene entonces protección constitucional ... [l]a Corte considera que hay tres elementos centrales a ser considerados en situaciones de esta naturaleza, y que son: a) de un lado, la urgencia e importancia misma del tratamiento para los intereses del menor; b) de otro lado, la intensidad del impacto del tratamiento sobre la autonomía actual y futura del niño...".

25 Sobre el artículo 29 de la CDN, que consagra el derecho del niño a la educación, el respectivo Comentario General señala: "el artículo atribuye importancia al proceso por el que se ha de promover el derecho a la educación. Así pues, los 
la mayoría de edad. Por esa razón, si bien los padres pueden manejar los tiempos en su proceso de formación para la autonomía, es también su deber entregarles las herramientas para que, a partir de los 18 años, estén en condiciones para ejercer sus derechos.

La educación sexual de los hijos e hijas, por el carácter íntimo que tiene la vida sexual, debe respetar su derecho de privacidad. A medida que van creciendo, son mayores los espacios de privacidad que padres y madres deberán respetar. En la adolescencia, necesariamente eso implica que ellos no pueden tener un conocimiento pleno sobre la conducta sexual de sus hijos e hijas. No sólo porque esa pretensión es ilusoria en los hechos, sino porque jurídicamente es inexigible. Esto no obsta, naturalmente, a que los padres aconsejen, enseñen, e incluso a que establezcan normas aplicables en materia de sexualidad.

La resolución que permite la distribución confidencial del anticonceptivo de emergencia a adolescentes puede justificarse bajo dos supuestos alternativos. O bien las jóvenes que tienen vida sexual están preparadas para el ejercicio de la autonomía que esa vida sexual implica, caso en el cual también estarían preparadas para tomar la decisión de consumir el anticonceptivo de emergencia. O bien las jóvenes están experimentando vida sexual sin tener las condiciones de madurez suficiente, caso en el cual su acceso al anticonceptivo de emergencia resguarda su autonomía futura al prevenir un embarazo para el cual tampoco están preparadas. En este segundo supuesto el Estado estaría ejerciendo un rol subsidiario al de los padres, porque la educación que éstos dieron a la niña no impidió que ella tuviera relaciones sexuales ${ }^{26}$.

Estas justificaciones se fundan, a su vez, en un supuesto empírico. Sin la garantía de la confidencialidad, las jóvenes que tienen sexo desprotegido no acudirían a los consultorios. Si esta premisa es correcta, la pérdida por parte de los padres de la valiosa oportunidad de aconsejar a sus hijas en el momento crítico en que corren el riesgo de embarazarse, en realidad no es tal. Sin la garantía de la confidencialidad dicha oportunidad nunca se presentaría, simplemente porque ninguna niña se atrevería a solicitar el fármaco.

La educación sexual de las hijas e hijos está entregada preferentemente a los padres. Es un proceso que involucra ir ofreciéndoles herramientas y oportunidades para ejercer su autonomía progresivamente hasta su mayoría de edad, en que el derecho les reconoce un goce pleno de ella. Respecto de las hijas, los padres tienen el especial desafío de educarlas para vivir autónomamente en una sociedad que discrimina y desconoce derechos incluso a las mujeres adultas.

Concebir el derecho de los padres como contrario u opuesto a la autonomía de los hijos e hijas supone no comprender de qué se trata la obligación de educar.

valores que se inculcan en el proceso educativo no deben socavar, sino consolidar, los esfuerzos destinados a promover el disfrute de otros derechos", CRC/GC/2001/1, 17 de abril de 2001.

26 Véase Comité de los Derechos del Niño, Observación General № 4 (2003), "La salud y el desarrollo de los adolescentes en el contexto de la Convención sobre los Derechos del Niño", especialmente los párrafos 11, 28 y 32 que tratan sobre derecho de los y las jóvenes a la salud reproductiva y confidencialidad. 